\title{
Constraining the $\Lambda$-nucleus potential within the Liège intranuclear cascade model
}

\author{
J. L. Rodríguez-Sánchez, ${ }^{1,2}$ J.-C. David, ${ }^{3}$ J. Hirtz, ${ }^{3,4}$ J. Cugnon, ${ }^{5}$ S. Leray, ${ }^{3}$ and D. Mancusi ${ }^{6}$ \\ ${ }^{1}$ Universidad de Santiago de Compostela, E-15782 Santiago de Compostela, Spain \\ ${ }^{2}$ GSI-Helmholtzzentrum für Schwerionenforschung GmbH, D-64291 Darmstadt, Germany \\ ${ }^{3}$ IRFU, CEA, Université Paris-Saclay, F-91191 Gif-sur-Yvette, France \\ ${ }^{4}$ Center for space and habitability, Universität Bern, CH-30 12 Bern, Switzerland \\ ${ }^{5}$ AGO department, University of Liège, allée du 6 août 19, bâtiment B5, B-4000 Liège, Belgium \\ ${ }^{6}$ SERMA, CEA, Université Paris-Saclay, F-91191 Gif-sur-Yvette, France
}

( Dated: June 8, 2018)

\begin{abstract}
The new version of the Liège intranuclear cascade model INCL, recently extended to the production of strange particles and hypernuclei, is used to investigate the $\Lambda$-nucleus potential in a broad range of nuclear masses from $\mathrm{Si}$ to $\mathrm{Pb}$. The combination of INCL calculation results with experimental cross sections of $\Lambda$ hypernuclei, obtained from $\left(\pi^{+}, K^{+}\right)$reaction studies, allows us to constrain the $\Lambda$-nucleus potential depth with an accurancy of about $0.9 \mathrm{MeV}$. Our results show that the potential depth increases with the mass number $(\mathrm{A})$ from $28 \mathrm{MeV}$, in the region of medium-mass hypernuclei, up to a maximum of $39.6 \mathrm{MeV}$ in the region of heavy hypernuclei around $Z=82$. This deviation could be related with the nucleon-isospin dependence of the three-body $\Lambda$-nucleon-nucleon force in asymmetry matter whose contribution is more relevant in hyper-neutron matter due to a strong contribution from $\Lambda n n$ interactions.
\end{abstract}

PACS numbers: $21.80 .+\mathrm{a}$

Experimental study of hypernuclei is one of the few possibilities to obtain information about nucleonhyperon (NY), hyperon-hyperon (YY), and hyperonnucleon-nucleon (YNN) forces, which have a high impact in astrophysical applications [1-4]. Since some years NY potentials fitted to the existing experimental data on elastic NY scattering are available for the theoretical description of hypermatter and hypernuclei [5]. Unfortunately, since the experimental data is rather scarce in comparison with the nucleon-nucleon case, the corresponding potentials are not very constrained [6-8]. In the case of $\mathrm{YY}$ and $\mathrm{YNN}$ forces the situation is even more complicated because there are no available data.

Experimental data from emulsion studies provided the first measurements of binding energies for light hypernuclei [9]. These experimental findings were followed by many theoretical studies whose aim was to determine the potential well depth of $\Lambda$ particles in nuclear matter. On the one hand, in the first phenomenological studies based on a Woods-Saxon potential well, it was found that a potential depth of about $30 \mathrm{MeV}$ could provide a reasonable description of the data [10]. Subsequent phenomenological investigations showed that a multi-parameter fit of binding energies changing the nuclear radius and the $\Lambda$ nucleus potential gives a better agreement, obtaining a potential well with a depth of about $28 \mathrm{MeV}[11,12]$. On the other hand, from more sophisticated models based on Brueckner-Hartree-Fock [7], skyrme-Hartree-Fock [13], or soft-core one-boson-exchange [6] approaches the potential depth could fluctuate between 21 and $35 \mathrm{MeV}$. However, these studies were only carried out for light and medium-mass hypernuclei because of the scarce measurements of heavy hypernuclei.

In last decades, the strangeness-exchanging reactions $\left(\pi^{+}, K^{+}\right)$and $\left(K^{-}, \pi^{-}\right)$were also utilized for spectro- scopic study of hypernuclei using the excitation spectra to extract information about the $\Lambda$ shell structure, obtaining the binding energies of $\Lambda$ single-particle states with a resolution below $2 \mathrm{MeV}$ [14-18]. In the case of $\left(\pi^{+}, K^{+}\right)$reactions, the cross sections were also determined with an accurancy below $7 \%$.

In this work, we propose to study the $\Lambda$-nucleus potential depth by combining experimental data of hypernuclei produced in strangeness-exchanging $\left(\pi^{+}, K^{+}\right)$ reactions with theoretical calculations performed with sophisticated dynamical reaction models based on the intranuclear-cascade (INC) approach [19-23]. In these models, the reaction is described by means of a twostep process usually applied in spallation, fragmentation, and charge-exchange reactions [24-26]: the collision itself, where part of the nucleons contained in the target nucleus are removed or modified and some excitation energy and angular momenta are gained by the remnant; and subsequent de-excitation processes by evaporation of particles or, if applicable, by fission. Here, INC models are considered as a Monte Carlo method to solve numerically the dynamic transport equations describing the hadron-nucleus collision. The nature of INC models is essentially classical, being assumed that nucleons are perfectly localised in phase space and that they are bound by a potential. In this approach, the nuclear collision is treated as successive relativistic binary hadron-hadron collisions separated in time, where the positions and momenta of hadrons are followed as time evolves. It is also assumed that hadrons move along straight trajectories until they undergo a collision with another hadron or until they reach the surface, where they could eventually escape. Cross sections are determined from a set of collision events taken at different impact parameters and for which nucleon positions and momenta are initially sam- 
pled for each participant nucleus.

For our purpose, we use the Liège intranuclear-cascade model (INCL) [27] that has been recently extended towards high energies $(\sim 15 \mathrm{GeV})$ including multipion production [28], strange particles like kaons and hyperons [29], and the production of $\eta$ and $\omega$ mesons [30]. This new version of INCL permits us to predict the formation of hyperremnants and their characterization in atomic, mass, and strange numbers together with their excitation energies and angular momenta. These improvements in INCL also require de-excitation models considering the emission of hyperons, in particular, the evaporation of $\Lambda$ particles. Currently, there are a few number of deexcitation models that treat the evaporation of hyperons and the formation of hypernuclei. In this work we use the evaporation model ABLA07 developed at GSI by Schmidt and collaborators [31], recently extended to hypernuclei by us including the evaporation of $\Lambda$ particles on basis of Weisskopf's approach according to Ref. [32].

In the new version of INCL elementary cross sections related with the production and interaction of strange particles (such as $K, \bar{K}, \Sigma$, and $\Lambda$ ) were implemented using sophisticated parametrizations of available experimental data as well as their characteristics: angular distributions, momenta, and charge repartition of the particles in the associated final states [29]. These new ingredients made INCL become in a powerful tool to study the production of strange particles in nuclear matter and to go further in the understanding of hypernuclei formation.

Target density profiles are prepared at the first step of the simulation assuming independent Woods-Saxon density distributions for protons and neutrons according to the method described in Ref. [33]. For the WoodsSaxon density distribution the radius $\left(R_{0}\right)$ and the diffuseness parameter $(a)$ are taken from Hartree-FockBogoliubov calculations since they provide a good description of single-nucleon knockout reactions [34]. The initial nucleon momenta are uniformly distributed in hard Fermi spheres of radii $(2 Z / A)^{1 / 3} p_{F}$ for protons and $(2 N / A)^{1 / 3} p_{F}$ for neutrons (see Ref. [35] for details). Moreover, the nucleons are sampled in phase space taking into account the correlations between kinetic energy and radius of the potential well [33]. The relation is such

TABLE I. Nuclear radii (in fm) for neutrons, protons, and $\Lambda$ s used in INCL to define the Woods-Saxon potential well of the nuclei investigated in this work, as well as the separation energies (in $\mathrm{MeV}$ ) used in the de-excitation process.

\begin{tabular}{ccccccc}
\hline \hline Nucleus & $\mathrm{R}_{n}$ & $\mathrm{R}_{p}$ & $\mathrm{R}_{\Lambda}$ & $\mathrm{S}_{n}$ & $\mathrm{~S}_{p}$ & $\mathrm{~S}_{\Lambda}$ \\
\hline${ }^{28} \mathrm{Si}_{\Lambda}$ & 3.25 & 3.29 & 3.57 & 16.00 & 10.30 & 16.0 \\
${ }^{89} \mathrm{Y}_{\Lambda}$ & 5.01 & 4.84 & 5.13 & 9.55 & 6.51 & 22.1 \\
${ }^{139} \mathrm{La}_{\Lambda}$ & 5.85 & 5.83 & 5.93 & 7.04 & 5.87 & 23.8 \\
${ }^{208} \mathrm{~Pb}_{\Lambda}$ & 6.76 & 6.64 & 6.76 & 5.88 & 7.74 & 26.5 \\
\hline \hline
\end{tabular}

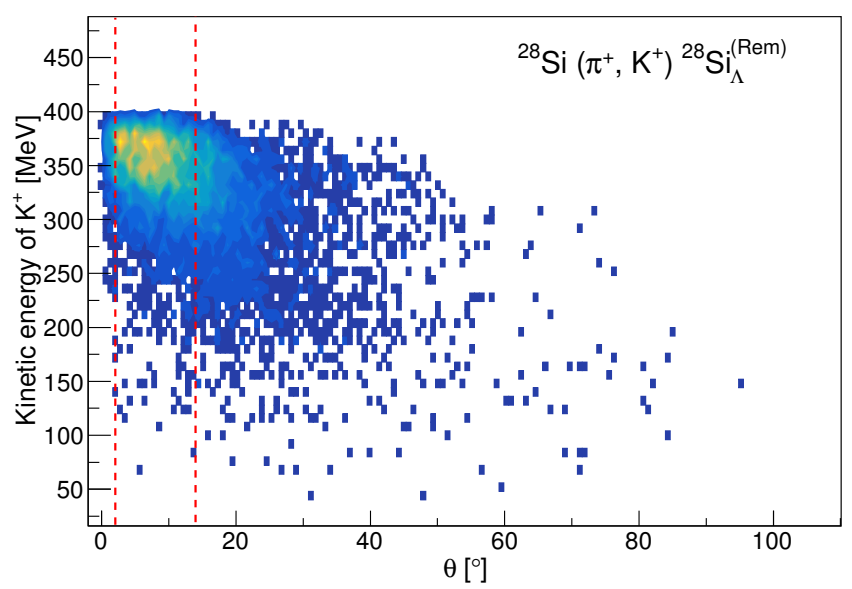

FIG. 1. (Color online) Scatter plot of the INCL predictions for the kinetic energy of $K^{+}$particles emitted in the production of hyperremnants ${ }^{28} \mathrm{Si}_{\Lambda}$ as a function of the angle $\theta$ for the reaction $\pi^{+}(1.06 \mathrm{GeV} / \mathrm{c})+{ }^{28} \mathrm{Si}$. The vertical lines located at $2^{\circ}$ and $14^{\circ}$ are to indicate the region selected for the comparison with experimental data.

that the space density distribution is given by the WoodsSaxon distribution. For $\Lambda$ particles we also use a WoodsSaxon potential well, taking the diffuseness parameter $\left(a_{\Lambda}\right)$ as for neutrons and a nuclear radius $R_{0}^{\Lambda}=(1.128+$ $\left.0.439 A^{-2 / 3}\right) A^{1 / 3}$ since this parametrization provides a reasonable description of the distance between hypernuclei binding energies [11]. In Table I are summarized the nuclear radii utilized to define the Woods-Saxon potential well of neutrons, protons, and $\Lambda$ particles. Finally, the $\Lambda$-nucleus potential well is set to a constant value of $28 \mathrm{MeV}$ by default, although it will be modified latter to constrain its depth within the strangeness-exchanging $\left(\pi^{+}, K^{+}\right)$reactions.

For the dynamical description of the collision between projectile and target nuclei, each event is fired at a given impact parameter $b$, ranging from 0 to a distance $b_{\max }$ given by the maximum radius of the target nucleus. If two hadrons approach each other at a distance lower than a minimum distance, they interact. The minimum distance is calculated from energy-dependent parametrizations of the hadron-hadron interaction cross sections for all possible collisions according to Ref. [36]. During the cascade process, the particles are divided into participants and spectators. Participants are defined as particles that have collided with at least one other participant, while spectators are the other particles. Collisions between spectators are forbidden in order to eliminate the spontaneous boiling of the Fermi sea, which leads to particles that could escape from the target, even if the particle is left alone. This condition is used because the spontaneous boiling is a direct violation of the Pauliexclusion principle. For nucleons, a strict Pauli blocking is also applied to the first collision to account for surface effects and for effects of the depletion of the Fermi sea [37]. For the subsequent collisions, we apply the Pauli 

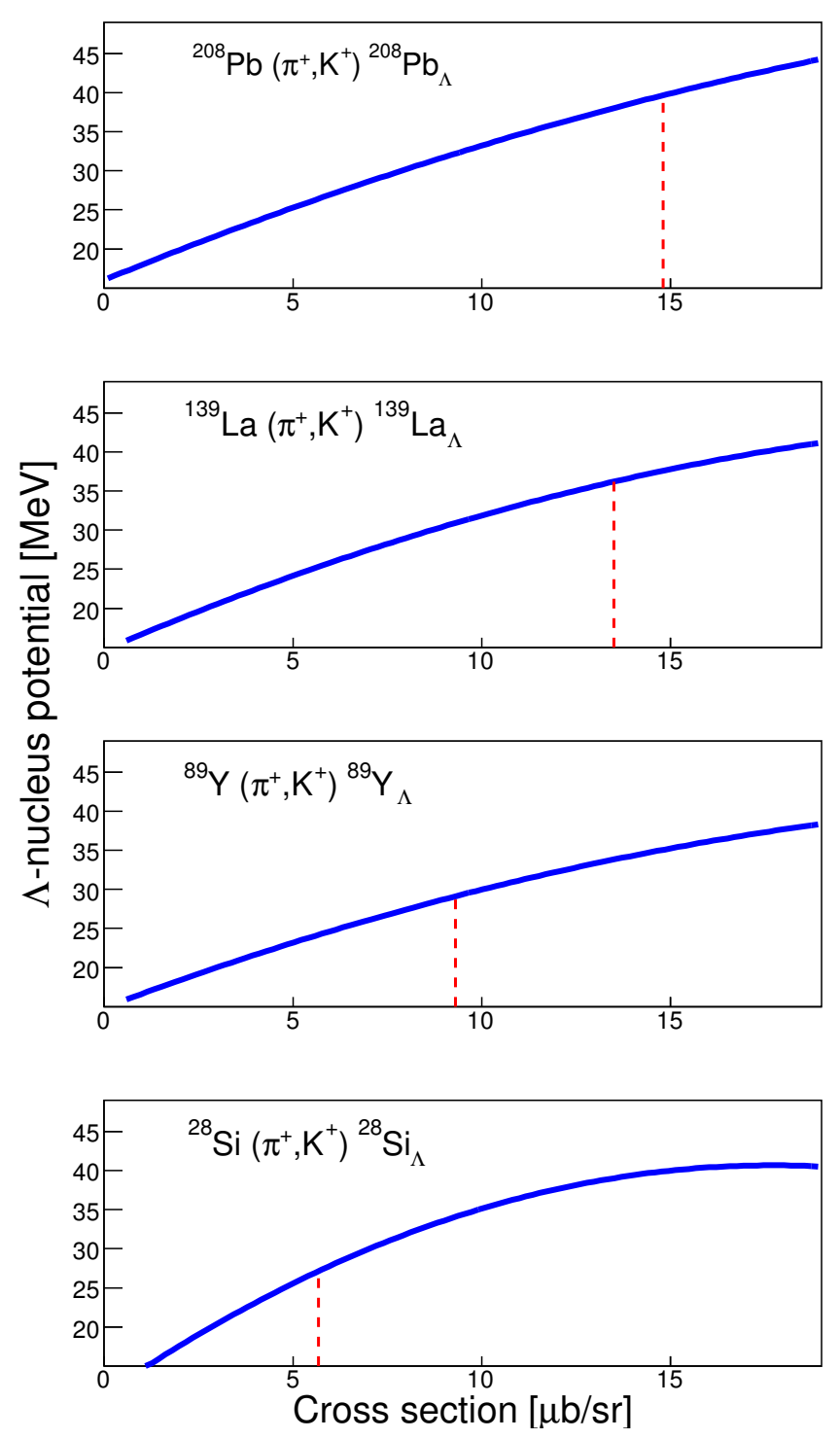

FIG. 2. (Color online) Correlation between the $\Lambda$-nucleus potential and the cross section of hypernuclei produced in the reactions indicated in each pannel for a pion momentum of $1.06 \mathrm{GeV} / \mathrm{c}$. The vertical lines indicate the experimental cross sections, obtained from Ref. [16], that were used to determine the potential well depth.

principle according to the usual procedure by means of statistical blocking factors, which do not have any effect in the case of single- $\Lambda$ hypernuclei. In addition, a consistent dynamical Pauli blocking is applied to all particles at the end of the cascade process to reject unphysical results, see Ref. [33] for more details. The excitation energy of the remnants is calculated from energy and momentum conservation laws applied over all existing particles in the initial configuration and at the end of the cascade process [33].

In Fig. 1 we display the kinetic energy of the positive kaons emerged from the reaction $\pi^{+}(1.06 \mathrm{GeV} / \mathrm{c})+{ }^{28} \mathrm{Si}$ producing hyperremnants of ${ }^{28} \mathrm{Si}_{\Lambda}$, this means that in this picture we do not take into account the de-excitation stage in which the excited hyperremnant could evaporate any particle. Here, the vertical lines indicate the angular selection that we will apply for the comparison with experimental data since the kaon emission angle is not affected by the de-excitation process.

For modeling the de-excitation stage, we use the ABLA07 code [31] that describes the de-excitation of a nucleus emitting $\gamma$-rays, neutrons, light-charged particles, and intermediate-mass fragments according to Weisskopf's formalism [38]. For a more realistic description of this process, the separation energies are taken from the atomic mass evaluation of 2016 [39] and the emission barriers for charged particles are determined with the Bass potential [40]. This model has been extended by us to account for the emission of $\Lambda$ particles and the production of cold hypernuclei following Refs. [32, 41]. The $\Lambda$ separation energies are parametrized according to the fit of experimental binding energies described in Ref. [42]. In addition, neutron and proton separation energies are modified in case of hypernuclei to take into account the hyperenergy released from the presence of the $\Lambda$ particle [41]. The values of all the separation energies used in this work are also listed in Table I. For this specific work, we do not need to introduce more ingredients in the de-excitation process since we are only interested in the survival probability of the isobaric hyperremnant that is determined basically by the number of excited hyperremnants with an excitation energy that does not surpass the minimum particle separation energy, defined as $\mathrm{S}_{\text {min }}=$ $\min \left\{\mathrm{S}_{n}, \mathrm{~S}_{p}, \mathrm{~S}_{\Lambda}\right\}$. This probability can be written as

$$
P_{E^{*} \leq S_{\min }}=\frac{1}{N} \sum_{j=0}^{N} \Theta\left(S_{\min }-E_{j}^{*}\right),
$$

where $\Theta$ is the Heaviside function and $N$ the total num-

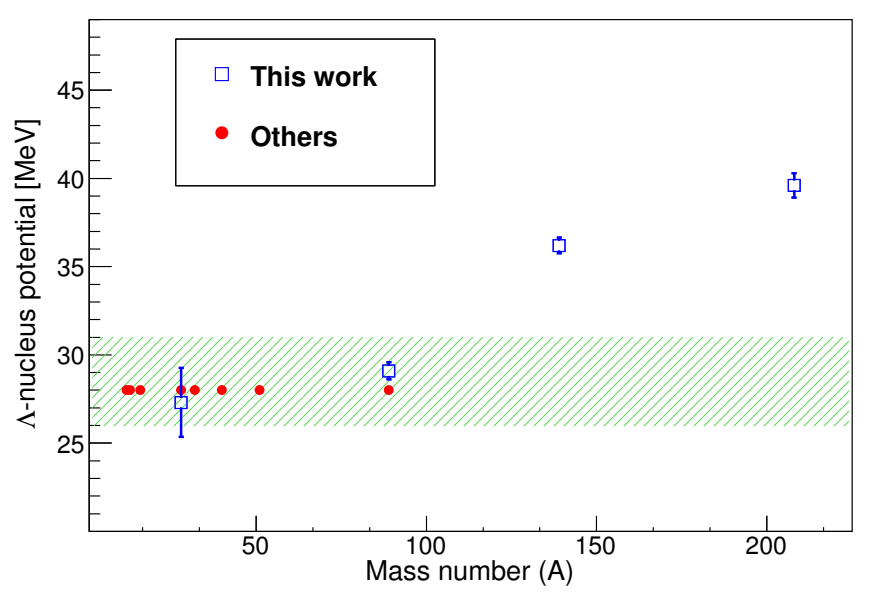

FIG. 3. (Color online) $\Lambda$-nucleus potential obtained from our model calculations (open squares) compared with the values reported in other works (solid circles). Dashed area is just to show the expected region for the $\Lambda$ potential depth. 
ber of isobaric hyperremnants produced by strangenessexchanging reactions.

In Fig. 2 we show the results obtained from our calculations for the strangeness-exchanging reactions indicated in each pannel. In these pictures we illustrate how the cross section evolves with the $\Lambda$-nucleus potential depth. One can see that the cross sections increase with the $\Lambda$ potential, which is basically explained by the increase of the phase space compatible with the kinetic energy gained by the $\Lambda$ particle. After defining these correlations for each hypernucleus, we can use the experimental cross sections, indicated in the figure with vertical dashed lines, to constrain the $\Lambda$-nucleus potential depth.

Our findings for the $\Lambda$-nucleus potential depth (open squares) are displayed in Fig. 3 together with the results obtained in other works. For hypernuclei with mass numbers below 89 we obtain a potential depth of around $28 \mathrm{MeV}$ that is in agreement with the values reported in other works based on phenomenological approaches [11] and on Skyrme-Hartree-Fock calculations [13]. However, for higher masses we find a potential depth of $36.2 \mathrm{MeV}$ for ${ }^{139} \mathrm{La}_{\Lambda}$ and $39.6 \mathrm{MeV}$ for ${ }^{208} \mathrm{~Pb}_{\Lambda}$, which were not observed in previous investigations because of the scarce experimental measurements in the region of heavy hypernuclei. We also performed INCL calculations assuming a smaller phenomenological radius of $R_{0}^{\Lambda}=1.165 A^{1 / 3}$ suggested by Gal and collaborators [43], which leads to a lower $\Lambda$ potential depth for $\mathrm{Pb}$ of about $37 \mathrm{MeV}$, but this value is still far from being adequate according to other theoretical predictions and thus it cannot explain the observed deviation. Recently, some works have also pointed out this possible deviation [8] whose understanding is still not clear.

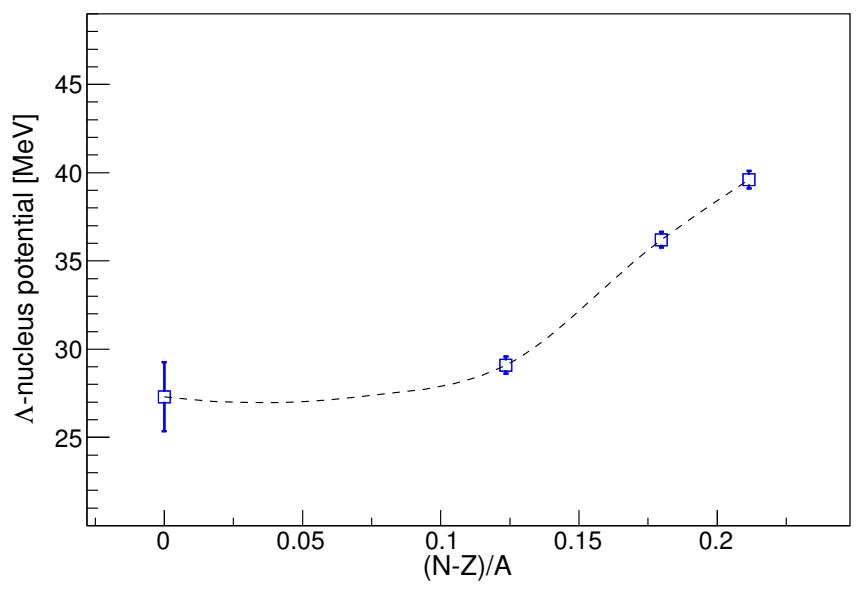

FIG. 4. (Color online) $\Lambda$-nucleus potential obtained from our approach as a function of the asymmetry $(N-Z) / A$. The dashed line is just a guide to the eye.
To go further, in Fig. 4 we also display the $\Lambda$-nucleus potential depth as a function of the asymmetry of the target nucleus, defined as $(N-Z) / A$, where $N$ is the number of neutrons, $Z$ is the number of protons, and $A$ is the mass number. This asymmetry parameter was proposed recently to study the nucleon-isospin dependence of the three-body $\Lambda N N$ force in neutron-rich hypermatter that could be important for the prediction of neutron star properties [44]. In the picture, we can observe a strong increase of the $\Lambda$ potential depth with this parameter when we move towards neutron-rich nuclei, which have a large neutron abundance at the surface that enhances the $\Lambda n n$ interaction. Note that in our INCL calculations we do not take into account three-body collisions, but it is clearly evident that their effects are included in the experimental separation energies and cross sections that we use as an input in our approach. Therefore, the deviation that we find in Fig. 3 could be a first evidence of such dependence that should be investigated in detail.

In conclusion, a new version of the Liège intranuclear cascade model INCL has been used for the first time to investigate the production of hypernuclei in strangenessexchanging collisions. INCL calculations, together with experimental $\Lambda$-separation energies, are utilized to establish direct correlations between the cross sections of hypernuclei produced via peripheral strangenessexchanging reactions $\left(\pi^{+}, K^{+}\right)$and $\Lambda$-nucleus potential depth. Experimental cross sections are then used to determine the potential depth with an average accurancy of $0.9 \mathrm{MeV}$. These constraints provide a potential depth of about $28 \mathrm{MeV}$ for medium-mass hypernuclei that is consistent with the results obtained in other works, whereas for heavy hypernuclei around $\mathrm{Pb}$, we obtain a potential depth of 39.6 MeV. This deviation was also pointed out previously, but without any clear conclusion. To go a step further, we also investigate how the $\Lambda$-nucleus potential depth evolves with the asymmetry of the nucleus. This kind of study was suggested recently to investigate the fundamental problem of the nucleon-isospin dependence of the three-body $\Lambda N N$ interaction [44], which could be important in the study of neutron star matter due to a strong contribution from the $\Lambda n n$ interaction. Surprisingly, we find a strong increase of the $\Lambda$-nucleus potential depth when we move towards neutron-rich nuclei, concluding that this fact could be a first proof of such strong interaction. We also think that more measurements in the region of heavy hypernuclei and theoretical analysis should be performed in order to confirm and understand this interesting behavior.

We wish to thank Dr. Alain Boudard for enlightening discussions and Dr. Georg Schnabel for his technical support. This work has been partially supported by the Department of Education, Culture and University Organization of the Regional Government of Galicia under the program of postdoctoral fellowships and by the EU ENSAR2 FP7 project (grant agreement 654002). 
[1] D. Lonardoni, A. Lovato, S. Gandolfi, and F. Pederiva, Phys. Rev. Lett. 114, 092301 (2015).

[2] A. I. Qauli, M. Iqbal, A. Sulaksono, and H.S. Ramadhan, Phys. Rev. D 93, 104056 (2016).

[3] R. Wirth and R. Roth, Phys. Rev. Lett. 117, 182501 (2016).

[4] M. Fortin, S. S. Avancini, C. Providência, and I. Vidaña, Phys. Rev. C 95, 065803 (2017).

[5] K. Tominaga, T. Ueda, M. Yamaguchi, N. Kijima, D. Okamoto, K. Miyagawa, T. Yamada, Nucl. Phys. A 642, 483 (1998).

[6] Th. A. Rijken, V. G. J. Stoks, and Y. Yamamoto, Phys. Rev. C 59, 21 (1999).

[7] H.-J. Schulze, M. Baldo, U. Lombardo, J. Cugnon, and A. Lejeune, Phys. Rev. C 57, 704 (1998).

[8] H.-J. Schulze and T. Rijken, Phys. Rev. C 88, 024322 (2013).

[9] D. H. Davis and J. Pniewski, Contemp. Phys. 27, 91 (1986).

[10] C. B. Dover, l. Ludeking, and G. E. Walker, Phys. Rev. C 22, 2073 (1980).

[11] D. J. Millener, C. B. Dover, and A. Gal, Phys. Rev. C 38, 2700 (1988).

[12] A. Bouyssy and J. Hüfner, Phys. Lett. 64B, 276 (1976).

[13] J. Cugnon, A. Lejeune, and H.-J. Schulze, Phys. Rev. C 62, 064308 (2000).

[14] R. Bertini et al., Phys. Lett. 83B, 306 (1979).

[15] R. Bertini et al., Nucl. Phys. A 360, 315 (1981).

[16] T. Hasegawa et al., Phys. Rev. C 53, 1210 (1996).

[17] P. H. Pile et al., Phys. Rev. Lett. 66, 2585 (1991).

[18] P. K. Saha et al., Phys. Rev. Lett. 94, 052502 (2005).

[19] H. W. Bertini, Phys. Rev. 131, 1801 (1963); Phys. Rev. 188, 1711 (1969).

[20] K. Chen, Z. Fraenkel, G. Friedlander, J. R. Grover, J. M. Miller, and Y. Shimamoto, Phys. Rev. 166, 949 (1968).

[21] Y. Yariv and Z. Fraenkel, Phys. Rev. C 20, 2227 (1979).

[22] V. S. Barashenkov, B. F. Kostenko, and A. M. Zadorogny, Nucl. Phys. A 338, 413 (1980).

[23] J. Cugnon, Phys. Rev. C 23, 2094 (1981).

[24] R. Serber, Phys. Rev. 72, 1114 (1947).

[25] A. Boudard, J. Cugnon, J.-C. David, S. Leray, and D. Mancusi, Phys. Rev. C 87, 014606 (2013).

[26] Th. Aoust and J. Cugnon, Phys. Rev. C 74, 064607 (2006).

[27] D. Mancusi, A. Boudard, J. Cugnon, J.-C. David, P. Kai- taniemi, and S. Leray, Phys. Rev. C 90, 054602 (2014).

[28] S. Pedoux, J. Cugnon, A. Boudard, J.-C. David, and S. Leray, Adv. Space Res. 44, 926 (2009).

[29] J. Hirtz, J.-C. David, A. Boudard, J. Cugnon, S. Leray, D. Mancusi, submitted to Eur. Phys. J. Plus.

[30] J.-C. David, A. Boudard, J. Cugnon, J. Hirtz, S. Leray, D. Mancusi, J. L. Rodríguez-Sánchez, accepted in Eur. Phys. J. Plus.

[31] A. Kelić, M. V. Ricciardi, and K.-H. Schmidt, Proceedings of Joint ICTP-IAEA Advanced Workshop on Model Codes for Spallation Reactions, ICTP Trieste, Italy, 4-8 February 2008, edited by D. Filges, S. Leray, Y. Yariv, A. Mengoni, A. Stanculescu, and G. Mank (IAEA INDC(NDS)-530, Vienna, 2008), pp. 181-221.

[32] A. S. Botvina, N. Buyukcizmeci, A. Ergun, R. Ogul, M. Bleicher, and J. Pochodzalla, Phys. Rev. C 94, 054615 (2016).

[33] A. Boudard, J. Cugnon, S. Leray, and C. Volant, Phys. Rev. C 66, 044615 (2002).

[34] J. L. Rodríguez-Sánchez, J.-C. David, D. Mancusi, A. Boudard, J. Cugnon, and S. Leray, Phys. Rev. C 96, 054602 (2017).

[35] D. Mancusi, A. Boudard, J. Carbonell, J. Cugnon, J.-C. David, and S. Leray, Phys. Rev. C 91, 034602 (2015).

[36] J. Cugnon, D. L'Hôte, and J. Vandermeulen, Nucl. Instr. Methods Phys. Res., Sect. B 111, 215 (1996).

[37] J. Cugnon and P. Henrotte, Eur. Phys. J. A 16, 393 (2003).

[38] V. F. Weisskopf and D. H. Ewing, Phys. Rev. 57, 472 (1940).

[39] W. J. Huang, G. Audi, M. Wang, F. G. Kondev, S. Naimi, and X. Xu, Chin. Phys. C 41, 030002 (2017).

[40] R. Bass, Proceedings of the Symposium on Deep-Inelastic and Fusion Reactions with Heavy Ions, Springer Verlag, Berlin, 1980.

[41] N. Buyukcizmeci, A. S. Botvina, J. Pochodzalla, and M. Bleicher, Phys. Rev. C 88, 014611 (2013).

[42] C. Samanta, P. Roy Chowdhury, and D. N. Basu, J. Phys. G.: Nucl. Part. Phys. 32, 363 (2006).

[43] A. Gal, E. V. Hungerford, and D. J. Millener, Rev. Mod. Phys. 88, 035004 (2016).

[44] D. Lonardoni and F. Pederiva, https://arxiv.org/abs/1711.07521. 